\title{
The Nucleolin Antagonist N6L Inhibits LINEI Retrotransposon Activity in Non-Small Cell Lung Carcinoma Cells
}

\author{
Kenneth S. Ramos ${ }^{1,2}$, Sara Moore², Isabel Runge², Marco A. Tavera-Garcia², Ilaria Cascone ${ }^{3}$, Jose Courty ${ }^{3}$, \\ Elsa M. Reyes-Reyes $2,4 \llbracket$ \\ 1. Texas A\&M University College of Medicine, Department of Medicine and Institute of Biosciences and Technology, Houston, Texas 77030, USA. \\ 2. University of Arizona College of Medicine, Department of Medicine, Division of Pulmonary, Allergy, Critical Care, and Sleep Medicine, Tucson, Arizona \\ 85721. \\ 3. University of Paris Est (UPEC), ERL-CNRS 9215, Laboratory of Growth, Reparation, and Tissue Regeneration (CRRET), UPEC, 94010 Créteil, France. \\ 4. Department of Cellular and Molecular Medicine, University of Arizona Cancer Center, Tucson, AZ, 85721. \\ $\square$ Corresponding author: Elsa Merit Reyes-Reyes, Ph.D., Department of Medicine, Division of Pulmonary, Allergy, Critical Care, and Sleep Medicine, \\ University of Arizona College of Medicine, 1656 E. Mabel St., Tucson AZ 85724 Phone: 5206262871 Email: emreye01@email.arizona.edu
}

() The author(s). This is an open access article distributed under the terms of the Creative Commons Attribution License (https://creativecommons.org/licenses/by/4.0/). See http:/ /ivyspring.com/terms for full terms and conditions.

Received: 2019.06.20; Accepted: 2019.09.18; Published: 2020.01.01

\begin{abstract}
Lung cancer is the most common cause of cancer death in the United States. The genome of nonsmall cell lung cancer (NSCLC), the most frequent lung cancer type, is strongly affected by Long Interspersed Nuclear Element (LINEI) insertions. Active LINEls are repetitive DNA sequences that can amplify themselves in the genome utilizing a retrotransposition mechanism whereby LINE1 is copied via reverse transcription and inserted at target sites. ORFIP and ORF2p are LINEI encoded proteins essential for LINEI retrotransposition. LINEIs are silenced epigenetically in somatic tissues, and their reactivation has been associated with cancer pathogenesis. Here, we present evidence that nucleolin (NCL) regulates expression of LINEI-ORFIP (LI-ORFIP) in NSCLC cells. Genetic knockdown of NCL significantly inhibited expression of LI-ORFlp in various NSCLC cell lines. Treatment with the investigational NCL antagonist N6L ablated L1-ORFlp expression in all cell lines constitutively expressing L1-ORFp. N6L displayed a stronger antiproliferative activity in NSCLC tumor cell lines expressing the highest LI-ORFlp protein levels. Moreover, N6L treatment of nude mice bearing NSCLC tumor xenografts blocked LI-ORFlp expression and effectively inhibited tumor growth. These data indicate that LI-ORFIP expression is regulated by $\mathrm{NCL}$ and identify NCL as a novel promising target for pharmacological inhibition of LINE1.
\end{abstract}

Key words: Nucleolin, LINE1, NSCLC, and Lung cancer.

\section{Introduction}

Non-small cell lung cancer (NSCLC) is the most common type of lung cancer and the leading cause of cancer-related mortality and economic burden in the United States [1]. More than $65 \%$ of NSCLC patients show cancer progression presenting with locally advanced or metastatic disease [2]. Current treatments have relatively low response rates and significant toxicity. Therefore, identification of new targets and drugs is needed to aid in the development of alternative therapeutic options for patients with
NSCLC [3].

LINE1s are autonomous and highly mutagenic genetic elements that mobilize throughout the genome via retrotransposition [4, 5]. In humans, LINE1 copies constitute 17 to $20 \%$ of the genome, though only $\sim 100$ LINE1s per individual remain retrotransposition competent due to truncations during the course of reverse transcription and polymorphic variations in LINE1 sequence. LINE1 encodes two proteins: L1-ORF1p, a $40 \mathrm{kDa}$ protein 
with nucleic acid binding activity, and ORF2p, a 150 $\mathrm{kDa}$ protein with endonuclease and reverse transcriptase activities [6]. A complete cycle of L1 retrotransposition consists of transcription of L1 RNA, export into the cytoplasm, translation of ORF1 and ORF2, association of L1 RNA with ORF1 and ORF2 proteins to form ribonucleoprotein (RNP) particles, import of RNPs into the nucleus, reverse transcription, and integration into new genomic locations [7]. Active LINE1s are a source of endogenous mutagenesis, with reactivation in somatic cells associated with a variety of genetic alternations including, aberrant splicing, exon skipping, gene fusions, and genome rearrangements that change gene expression and promote genomic instability [6].

The genome of NSCLCs is strongly affected by L1 insertions $[8,9]$. As such, systematic investigation of mechanisms responsible for the regulation of LINE1 protein expression and the specific roles of LINE1s in NSCLC progression are crucial to the identification of novel molecular targets for NSCLC therapy.

Nucleolin (NCL) is an RNA-binding protein, and more than $90 \%$ of NCL is localized in the nucleolus. However, NCL is also present in other cellular compartments, including the nucleoplasm, cytoplasm, and cell surface. NCL has multiple roles in ribosome biogenesis, transcription, DNA and RNA metabolism, DNA repair, and apoptosis [10-13]. In contrast to normal tissue, NCL excessively accumulates in the cytoplasm and the cell surface (extranuclear) of several cancer cell types, including lung cancer cells [14-17]. One key function of NCL is the modulation of cellular protein levels by binding mRNA targets to regulate RNA turnover and translation. NCL was recently reported to bind LINE1 RNA [18], but the functional implications of this interaction are not yet fully understood. Here, we present evidence that NCL modulates LINE1 activity by regulating the expression of L1-ORF1 protein. This interaction can be targeted pharmacologically by NCL antagonists, thus opening the door to novel therapies for lung cancer treatment.

\section{Methods and Materials}

\section{Materials}

NucAnt 6L (N6L) was synthesized as previously described and dissolved in 5\% D-mannitol [19]. AS1411 (5'-GGTGGTGGTGGTTGTGGTGGTGGTGG$3^{\prime}$ ) oligodeoxynucleotide in the desalted form was purchased from Life Technologies (Grand Island, NY). Anti-rabbit IgG and anti-mouse IgG antibodies linked to horseradish peroxidase (HRP), anti-nucleolin monoclonal (MS-3), and anti-GAPDH monoclonal antibody were from Santa Cruz Biotech. A custom made polyclonal anti-human L1-ORF1p antibody was produced by New England Peptide LLC. The antigen peptide "MGKKQNRKTGNSKTQ" sequence used to generate the rabbit polyclonal anti-human L1-ORF1p does not match the murine ORF1p amino acid sequence. Further, the specificity of the antibody against human L1-ORF1p was validated using several criteria including a single band of the expected molecular weight by Western blot, use of positive and negative control cell lines and tissue sources, specific knockdown of signal intensity using siRNAs, and high reproducibility between experimental runs and antibody lots. Biochemical validation of the anti-L1-ORF1p has been described previously [20].

\section{Cell cultures and treatments}

Cell lines were purchased from the American Type Culture Collection (ATCC). Cells were grown in a humidified environment at $37{ }^{\circ} \mathrm{C}$ with $5 \% \mathrm{CO}_{2}$. Cell lines were confirmed to be free of mycoplasma contamination (MycoAlert; Lonza). NSCLC cell lines (NCI-H460, NCI-H520, NCI-H1299, and A549) were grown in RPMI medium supplemented with fetal bovine serum at a concentration of $10 \%, 62.5 \mathrm{mg} / \mathrm{mL}$ penicillin and $100 \mathrm{mg} / \mathrm{mL}$ streptomycin (Life Technologies). The human bronchial epithelial cell lines BEAS-2B and BZR were grown in LHC-9 medium (Thermo Fisher Scientific). Verification of all cell line identities was performed by short tandem repeat (STR) sequencing using reference databases from ATCC (Genetics Core, University of Arizona, AZ). Cells were plated 1-day before treatments and challenged with the desired concentrations of N6L or AS1411 as indicated in figure legends. For biochemical analyses, cells were lysed with buffer containing $150 \mathrm{mmol} / \mathrm{L} \mathrm{NaCl}, 2 \mathrm{mmol} / \mathrm{L}$ EDTA, 50 $\mathrm{mmol} / \mathrm{L}$ Tris- $\mathrm{HCl}, \quad 0.25 \%$ deoxycholic acid, $1 \%$ IGEPAL CA-630 (pH 7.5), supplemented with protease and phosphatase inhibitor cocktails (EMD Millipore) for $5 \mathrm{~min}$ at $4^{\circ} \mathrm{C}$, and then cleared by centrifugation at $16,000 \times \mathrm{g}$ for 10 minutes at $4^{\circ} \mathrm{C}$. All protein concentrations were determined using the bicinchoninic acid assay (Thermo Fisher Scientific).

\section{Western blot analyses}

Total cell lysates were resolved by SDSpolyacrylamide gel electrophoresis and electrotransferred onto polyvinylidene fluoride membranes (Millipore) in Tris-glycine buffer containing $20 \%$ methanol. Proteins were detected by immunoblotting. Membranes were stripped of bound antibodies using Restore $^{\mathrm{TM}}$ PLUS Western Blot Stripping Buffer (Thermo Fisher Scientific) and 
reprobed as described in figure legends.

\section{Nucleolin knockdown expression}

NCL small interfering RNA (siRNA) duplex sequences [5'-GGUCGUCAUACCUCAGAAGtt (NCL\#1， ID\#144014)， 5'-CGGUGAAAUUGAUGG AAAUtt (NCL\#2, ID\#144016)] and scrambled siRNA sequences (Silencer Negative Control \#1 siRNA (AM4635)) were chemically synthesized and annealed by Life Technologies. BLAST analysis showed no homology to any sequence in the Human Genome Database, other than the intended target. siRNAs were transfected using Lipofectamine RNAiMAX (Life Technologies), according to manufacturer's directions. Briefly, cells were plated and incubated overnight to allow adherence, then transfected with siRNAs for $8 \mathrm{~h}$. Cell medium was replaced with fresh complete medium, and cells incubated for $72 \mathrm{~h}$ before analysis as described in figure legends.

\section{Cell proliferation}

Measurements of cell proliferation were completed using a 3-(4,5-dimethylthiazol-2-yl)-2, 5-diphenyltetrazolium bromide (MTT) assay [21]. This assay detects metabolic activity based on $\mathrm{NAD}(\mathrm{P}) \mathrm{H}$-dependent cellular oxidoreductase activity and has been used as a reliable measure of cell proliferation in cultured bronchial epithelial cells [20]. Briefly, 3,000 cells were seeded in quadruplicate 96-well plates and allowed to adhere overnight. Cells were treated with different concentrations of N6L and incubated for $72 \mathrm{~h}$ without changing the culture medium. The signal corresponding to medium from plates with no cells was subtracted as background. Cell proliferation was determined by normalizing to the proliferation of untreated cells for each cell type.

\section{Measurements of cell viability}

BEAS-2B and H520 cells $(60,000)$ were plated in 12-well plates one day before treatment. Cultures were either challenged with $10 \mu \mathrm{M}$ N6L or an equivalent volume of vehicle. Adherent and floating cells were harvested after three days and pelleted by centrifugation. The pellet was resuspended in $0.4 \%$ trypan blue/PBS solution and stained cells counted to measure viability.

\section{In vivo studies}

The Institutional Animal Care and Use Committee (IACUC) at the University of Arizona approved all experimental procedures involving animals. Healthy male, weanling nude mice (Fox1nu) were purchased from Charles River Laboratories Inc. After acclimation for a week in the animal facility, mice were injected subcutaneously with a single cell suspension consisting of $3 \times 10^{6} \mathrm{NCI}-\mathrm{H} 520$ cells in 200 $\mu \mathrm{L}$ PBS into each flank. When subcutaneous tumors reached a volume of approximately $100 \mathrm{~mm}^{3}$, the mice were randomized into two groups of 6 mice per group. The control group was given PBS and compared to animals given $10 \mathrm{mg} / \mathrm{kg} /$ day N6L dissolved in PBS three times per week by intraperitoneal injection. Tumor volume and body weights were recorded every two or three days for 16 days. Tumors were resected following euthanasia and processed for detection of L1-ORF1p expression by immunoblotting.

\section{Statistical analysis}

Experimental replicates were independent and performed on separate days. Comparisons between treated and control groups were carried out using multiple paired two-tailed t-tests or ANOVA followed by Tukey's multiple comparisons test as specified in figure legends. Statistical significance was denoted by $\mathrm{p}$-values less than 0.05 .

\section{Results}

\section{NCL regulates expression of LINEI}

Previous reports indicate that $50 \%$ of NSCLC have increased L1-ORF1p expression across a panel of different human lung neoplasms [9]. We have reported that stable ectopic overexpression of LINE1 in non-malignant human bronchial epithelial BEAS-2B cells induces oncogenic transformation and tumorigenesis in vivo, independent of its reverse transcriptase activity and active cycles of retrotransposition $[20,22]$. These findings suggest that LINE1 is involved in lung carcinogenesis and possibly serve as a novel candidate for targeted therapeutics during malignant progression of NSCLCs.

NCL modulates cellular protein levels by binding mRNA targets to control RNA turnover and translation. This protein is of interest given its ability to regulate cancer cell phenotypes and to partner with LINE1 RNA [18]. Therefore, studies were conducted to determine whether NCL modulates L1-ORF1p expression in NSCLC. We first examined the relative expression of L1-ORF1p and NCL in four NSCLC cell lines (NCI-H460, NCI-H520, NCI-H1299, and A549), compared to the non-malignant BEAS-2B cell line and its ras-transformed counterpart, BZR cells (Fig. 1A). Immunoblotting analysis showed that L1-ORF1p was strongly expressed constitutively in three NSCLC cell lines (NCI-H520>NCI-H1299>NCI-H460), while relative L1-ORF1p expression was detectable at low levels in BEAS-2B, BZR, and A549 cells (Fig. 1B and C). All tested cell lines showed strong expression of NCL (Fig. 1B and C). While the expression of L1-ORF1p did not consistently correlate with NCL 
expression (Fig. 1D), higher levels of NCL expression were preferentially observed in NSCLC cell lines with higher LINE1-ORFp1 expression (Fig. 1C).

Next, we examined whether NCL played a role in the regulation of LINE1 by examining the effect of genetic knocking down of NCL on L1-ORF1p expression in NCI-H520 cells. Immunoblot analyses confirmed that NCL expression could be reduced by $>90 \%$ in cells transfected with two distinct NCL siRNAs compared with cells transfected with scrambled siRNA (Fig. 1D). Knockdown of NCL elicited a dramatic decrease in L1-ORF1p expression (Fig 1E). These results indicate NCL is a positive regulator of L1-ORF1p expression.

\section{N6L, a NCL antagonist, inhibits LI-ORFIp expression}

To further evaluate the influence of NCL on L1-ORF1p expression, the next set of experiments was designed to determine if pharmacological agents that block NCL functions modulate expression of L1-ORF1p in NSCLC cells. Currently, N6L, a pseudopeptide, and AS141, a DNA aptamer, are the best options to study the biological functions of NCL. We analyzed L1-ORF1p expression profiles in NSCLC cells challenged with various concentrations of N6L and AS1411. N6L completely inhibited L1-ORF1p protein expression in NCI-H520, NCI-H460, and NCI-H1229 cells treated with $10 \mu \mathrm{M}$ for $24 \mathrm{~h}$ (Fig. 2A and B). In contrast, AS1411 was without effect in any of the cell lines (data not shown) or in NCI-H520 cells at high concentrations $(20 \mu \mathrm{M})$ (Fig. 2A). Because LINE1 can be reactivated under stress conditions, we tested the effects of N6L in low expressing BEAS-2B and BZR cells. N6L was without effect in these lines. These data indicate that L1-ORF1p expression is blocked by the NCL antagonist N6L and suggest that NCL may be a reasonable pharmacological target to inhibit LINE1-mediated progression of NSCLCs.

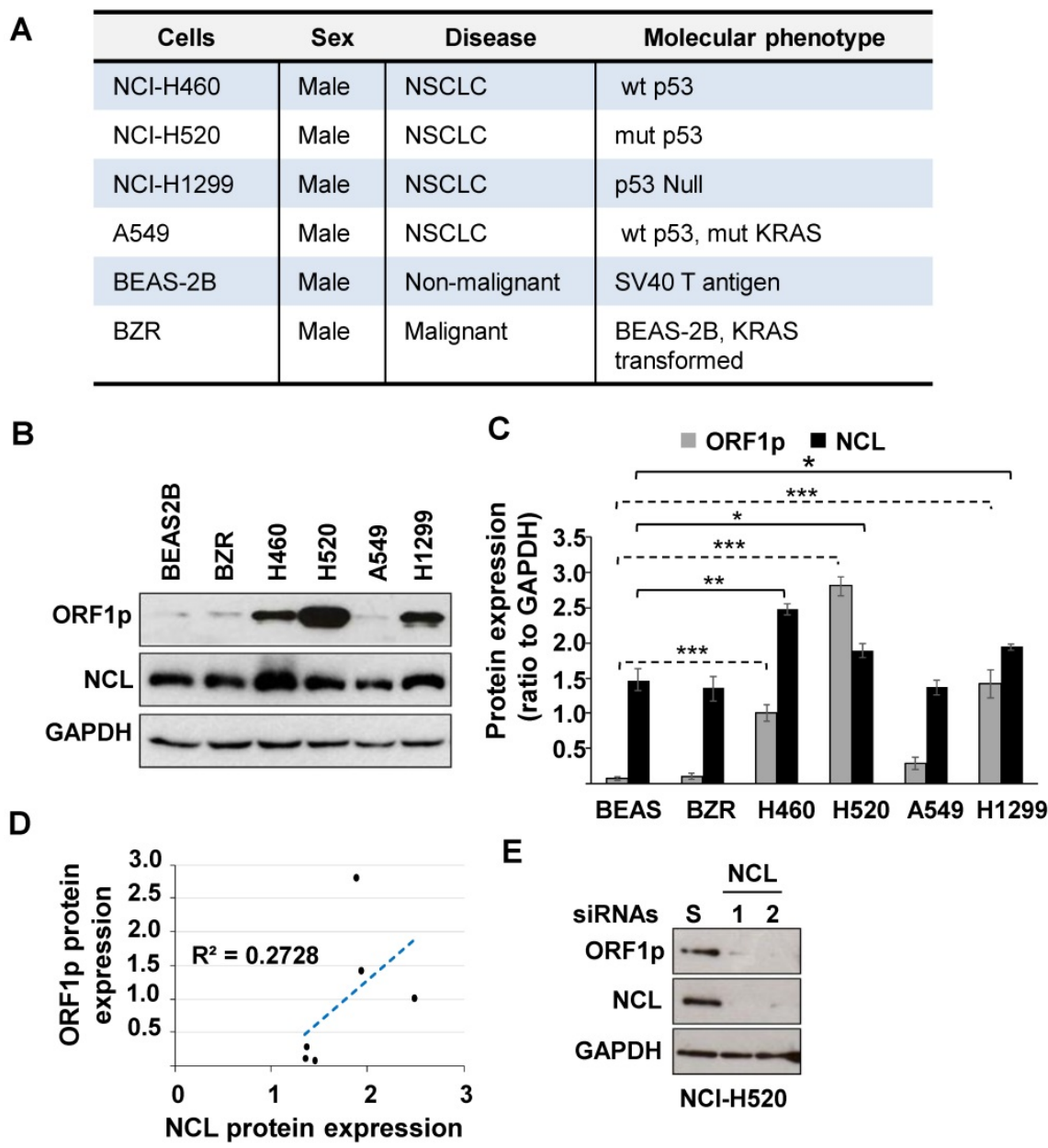

Figure 1: (A) Phenotypic profiles of lung cells employed in this study. (B) Whole-cell extracts from BEAS-2B, BZR, NCl-H460, NCl-H520, A549, or NCl-H1299 were examined by immunoblotting using L1-ORFlp, NCL, and GAPDH antibodies. (C) NCL, LI-ORFlp, and GAPDH were quantified by densitometry. Relative protein expression was expressed as NCL/GAPDH and L1-ORF1 /GAPDH ratios from three independent analyses. Error bars represent SEM. Statistical significance was determined using multiple paired two-tailed t-tests; $n=3 ;{ }^{*} p<0.05 ; * * p<0.001$, ** $p<0.0001$. (D) Correlation between L1-ORF1p and NCL protein levels. (E) $\mathrm{NCl}-\mathrm{H} 520$ cells were transfected with two different NCL siRNAs (NCL \#1 and 2). Three days post-transfection, cells were analyzed for expression of L1-ORFlp, $\mathrm{NCL}$, and GAPDH. 
A

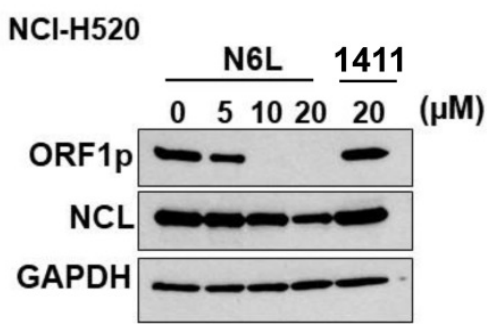

B

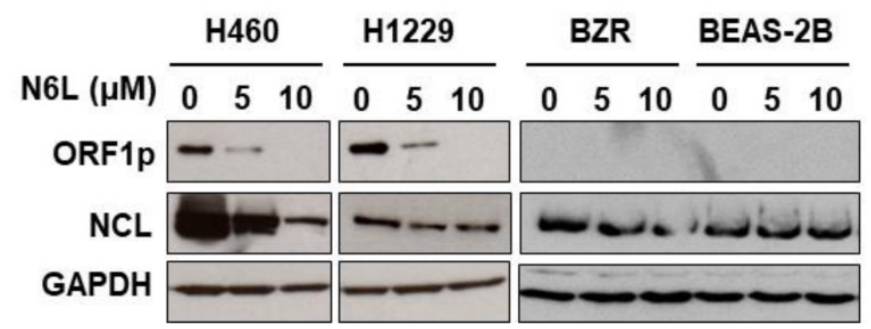

Figure 2: (A) NCl-H520 cells were treated with vehicle (5\% D-mannitol solution) or different concentration of N6L or $20 \mu \mathrm{M} \mathrm{AS1411}$ (AS) for 48h. (B) NCl-H460, $\mathrm{NCl}-\mathrm{H} 1299, \mathrm{BZR}$, and BEAS-2B cells were treated with different concentration of N6L for $48 \mathrm{~h}$. Whole-cell lysates were analyzed by immunoblotting using LI-ORFIp, NCL, and GAPDH antibodies.

A

B

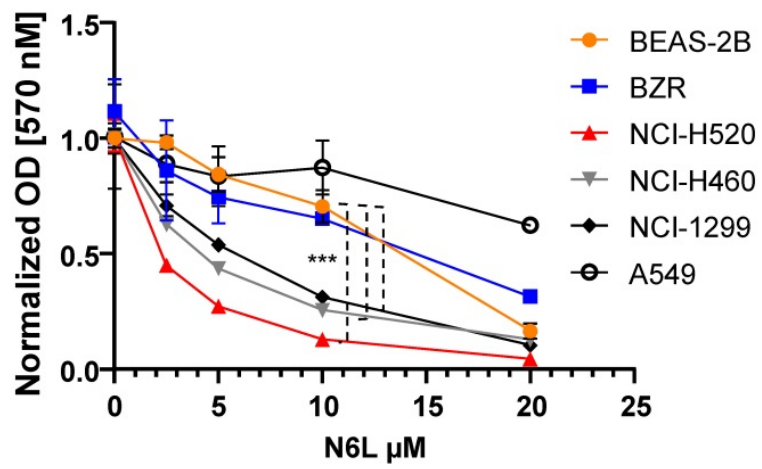

\begin{tabular}{|l|c|}
\hline \multicolumn{1}{|c|}{ Cells } & IC50 $\boldsymbol{\mu M}$ \\
\hline NCI-H520 & 2.2 \\
\hline NCI-H460 & 4.42 \\
\hline NCI-1299 & 5.97 \\
\hline BEAS-2B & 13.47 \\
\hline BZR & 14.82 \\
\hline
\end{tabular}

C

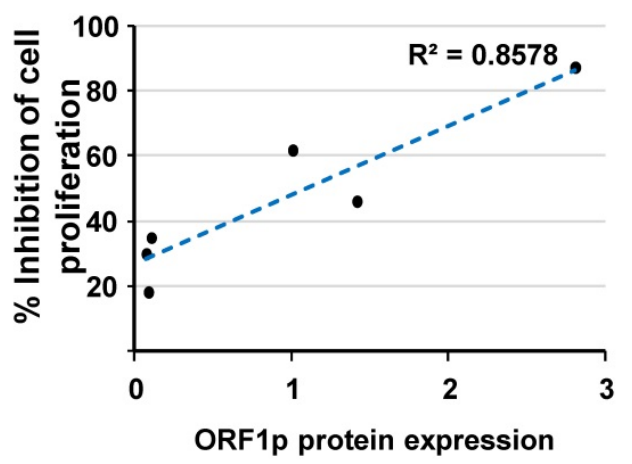

Figure 3: (A) Lung cells were treated with vehicle (5\% D-mannitol solution) or various concentrations of N6L for 3 days. The MTT assay was used to measure cell proliferation. Cell proliferation was determined by normalizing to the proliferation of untreated cells for each cell type. Error bars represent the SEM. Statistical significance was determined at $10 \mu \mathrm{M}$ N6L comparing BEAS $2 B$ versus $\mathrm{H} 520, \mathrm{NCl}-\mathrm{H} 460$ and $\mathrm{NCl}-\mathrm{H} 1299$ by ANOVA followed by Tukey's multiple comparisons test; $n=4 * * * p<0.0001$ (B) The half-maximal inhibitory concentration (IC50) of N6L was calculated after treatment for $72 \mathrm{~h}$. (C) Correlatio $\mathrm{n}$ between proliferation and L1-ORF1p protein levels.

\section{N6L activity correlates with LI-ORF1p expression}

N6L has potent growth inhibitory effects in several cell lines or primary cells derived from brain, breast, prostate, $\mathrm{T}$ lymphocytes, colon, pancreas, skin, and kidney carcinomas [23-25]. However, the effect of N6L on NSCLC cell growth has not being examined. Using the MTT assay as an indirect measure of cell proliferation we found that N6L preferentially inhibited NSCLC lines with the highest constitutive expression of L1-ORF1p (NCI-H520>NCI-H460>NCIH1299) compared to lines with lower expression (BEAS-2B, BZR and A549 cell lines) (Fig. 3A and B).

N6L $(10 \mu \mathrm{M})$ did not increase Trypan blue uptake in BEAS-2B or NCI-H520 cells after treatment for three days, indicating that NCL inhibition is associated with cytostatic effects, in the absence of cytotoxicity (not shown). Moreover, the antiproliferative activity of N6L correlated with the relative abundance of L1-ORF1p protein (Fig. 3C). These results suggest that the pharmacological effectiveness of N6L involves interference with L1-ORF1p expression.

\section{N6L inhibits L1-ORF Ip expression in vivo}

Given the inherent limitations of in vitro models, we next set out to determine whether the pharmacological efficacy of N6L was observed in vivo. To this end, nude mice with subcutaneous NCI-H520 xenografts received intraperitoneal injections of 250 $\mu \mathrm{g}$ of N6L in $200 \mu \mathrm{L}$ PBS per day three times a week for two consecutive weeks to approximate a cumulative dose of $10 \mathrm{mg} / \mathrm{kg} /$ day. Additional control groups received an equivalent amount of vehicle alone. Tumor volumes and body weights were recorded at regular intervals. Figure $4 \mathrm{~A}$ shows that N6L significantly inhibited tumor growth in the nude mouse xenograft model. No evidence of toxicity was observed for any of the treatments, as evidenced by the absence of changes in body weight (Fig. 4B), animal behavior, or organ examination at necropsy. Further, immunoblotting analysis showed that 
tumors from mice treated with N6L exhibited lower expression of L1-ORF1p compared to tumors from vehicle treated mice (Fig. 4C). These data indicate that the NCL antagonist N6L blocks LINE1 activity and tumor growth in vivo.

\section{Discussion}

Evidence is presented here that NCL positively regulates the expression of L1-ORF1p in NSCLC cell lines and that NCL may be targeted pharmacologically by N6L to regulate the oncogenic activity of LINE1 in NSCLC cell lines. L1-ORF1p is expressed constitutively in NSCLC tumors but not in healthy tissues $[8,9]$. In keeping with previous reports, we observed that L1-ORF1p was barely detected in non-malignant human bronchial epithelium cells BEAS-2B, but significantly increased under constituve conditions in the human NSCLS tumor cell lines, H460, H520, and H1299. Interestingly, the H460, H520, and H1299 cell lines also expressed higher levels of NCL than the non-malignant human bronchial epithelium cells BEAS-2B. NCL also increases murine L1-ORF2 expression by binding to its RNA in the internal ribosome entry site (IRES) to facilitate translation [26]. Consequently, NCL depletion decreases mouse L1

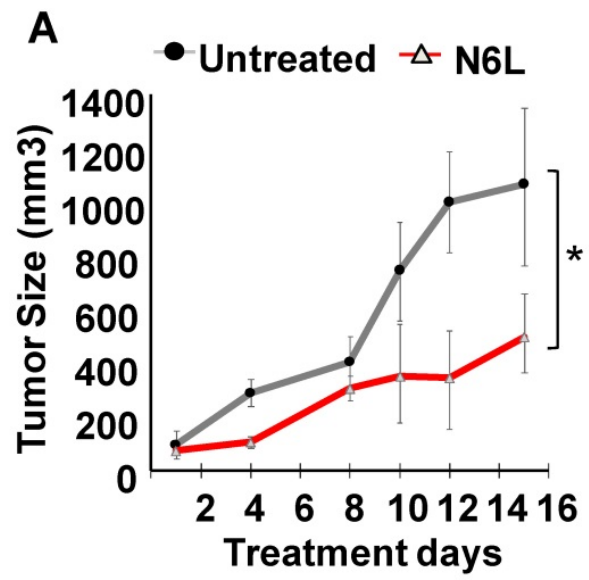

retrotransposition activity in vitro [26]. These findings support the conclusion that NCL can regulate oncogenic activity by influencing the expression and relative abundance of LINE1 proteins.

NCL plays significant roles in many physiological processes such as cellular proliferation, survival, and apoptosis [27]. Disruption of NCL homeostatic functions impairs cancer progression by altering signaling pathways through genetic and epigenetic mechanisms that control proliferation, survival, and metastasis of cancer cells [13]. Recently, NCL was shown to form a complex with LINE1 RNA and KAP1 (a transcriptional co-repressor). This complex promotes the self-renewal of embryonic stem cells by suppressing Dux expression, a master activator of the developmental transition program in two-cell embryos [18]. Thus, NCL may induce cancer progression by promoting cancer cell stemness via LINE1 RNA. Our previous findings showed that overexpression of LINE1 in non-malignant human bronchial epithelium cells induce oncogenic transformation and tumorigenesis in vivo [22]. Our present findings showing that NCL regulates L1-ORF1p expression suggests that dysregulation of NCL functions may promote lung carcinogenesis via LINE1 activation.

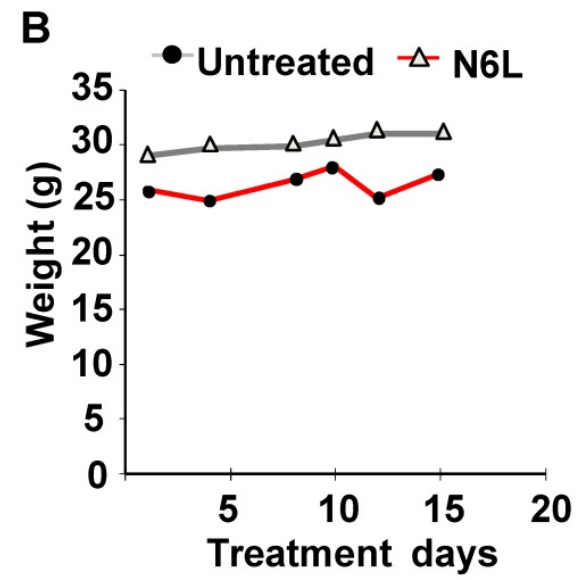

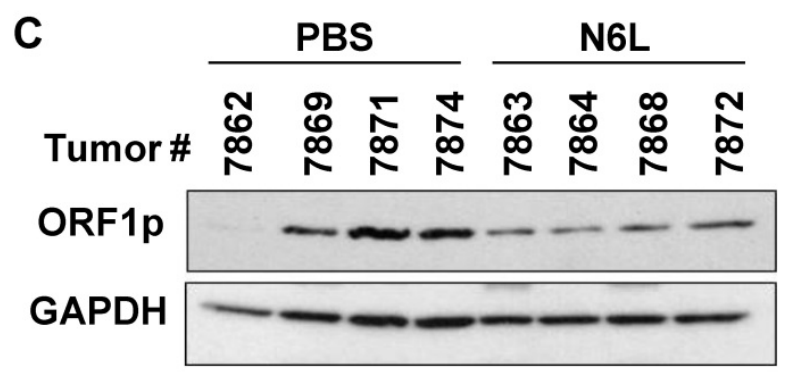

Figure 4: Subcutaneous tumors were established after injection of $3 \times 10^{6} \mathrm{NCl}-\mathrm{H} 520$ cells into 5 -week old male Nu/Nu mice. Mice were randomized into two groups and treated with either PBS or $10 \mathrm{mg} / \mathrm{kg} /$ day N6L in PBS three times per week given by intraperitoneal injection. (A) Tumor volume; (B) Body weights; (C) LINE-1 expression in tumor tissue measured by immunoblotting using L1-ORFIp, and GAPDH antibodies. Statistical significance (*p $=0.038)$ was determined by ANOVA followed by Tukey's multiple comparisons test. Error bars represent the SEM. 
N6L is a pseudopeptide that functions as a NCL antagonist through selective binding to NCL to inhibit tumor growth and angiogenesis [19, 23, 25, 28, 29]. N6L blocks survival signaling pathways, slows down cell cycle progression, induces autophagy, and inhibits tumor cell invasion [24, 25, 30,31]. Given that LINE1 increases cancer cell proliferation and tumorigenesis [20,32], the abilty of N6L to decrease L1-ORF1p expression in NSCLC cell lines and in vivo is consistent with observed impairments of NSCLC xenograft tumor growth. We conclude that the impact of N6L on tumor growth involves decreases in L1-ORF1p which in turn influence LINE1 oncogenic functions.

The finding that N6L, but not AS1411, blocked L1-ORF1p expression suggests that LINE1 interference by these agents is exerted via different mechanisms. N6L specifically binds to cell surface NCL to promote internalization through an endocytic mechanism. After internalization, N6L can be localized in the cytoplasm, nucleoplasm, and nucleolus [19], and is known to target both the nuclear and extranuclear functions of NCL [23, 24, 30, 33]. In contrast, AS1411 is a DNA aptamer that mainly internalizes into cells through the gulp of large vesicles using the micropinocytosis endocytic mechanism. Internalized AS1411 is localized only to the cytoplasm and never localizes to the nuclei of viable cells [34]. Thus, AS1411 only targets NCL extranuclear functions such as shuttling, signal transduction, or mRNA stability, which could also affect protein expression [35]. The observation that AS1411 did not affect L1-ORF1p expression suggests that nuclear functions of NCL are the ones involved in regulation of L1-ORF1p expression. It has been suggested that the antiproliferative activity of AS1411 is not specific for nucleolin targeting [36]. Thus, it is possible that AS1411 does not target key extranuclear functions of NCL linked to L1-ORF1p expression.

In summary, the ability of N6L to block the expression of L1-ORF1p suggests that LINE1 activity during NSCLC progression may be effectively regulated by pharmacological agents and amenable to precision therapeutics. N6L has already been tested in human clinical trials in other cancers, and clinical development efforts are ongoing. Therefore, it would be interesting to test whether N6L may be an effective therapeutic agent for NSCLC and whether the L1-ORF1p expression may serve as a biomarker to select patients for future $N 6 \mathrm{~L}$ treatment.

\section{Acknowledgements}

These studies were supported by funds from IRG-16-124-37 from America Cancer Society and University of Arizona Career Development Award to
EMRR, GURI Award to KSR, and the ANR-16 CE17-0023 from Agence National pour la Recherche to JC.

\section{Competing Interests}

The authors have declared that no competing interest exists.

\section{References}

1. Spira A, Halmos B, Powell CA. Update in Lung Cancer 2014. Am J Respir Crit Care Med. 2015; 192: 283-94.

2. Reck M, Heigener DF, Mok T, Soria JC, Rabe KF. Management of non-small-cell lung cancer: recent developments. Lancet. 2013; 382: 709-19.

3. Jonna S, Subramaniam DS. Molecular diagnostics and targeted therapies in non-small cell lung cancer (NSCLC): an update. Discov Med. 2019; 27: 167-70.

4. Cordaux R, Batzer MA. The impact of retrotransposons on human genome evolution. Nat Rev Genet. 2009; 10: 691-703.

5. de Koning AP, Gu W, Castoe TA, Batzer MA, Pollock DD. Repetitive elements may comprise over two-thirds of the human genome. PLoS Genet. 2011; 7: e1002384.

6. Beck CR, Garcia-Perez JL, Badge RM, Moran JV. LINE-1 elements in structural variation and disease. Annu Rev Genomics Hum Genet. 2011; 12: 187-215.

7. Ostertag EM, Kazazian HH, Jr. Biology of mammalian L1 retrotransposons. Annu Rev Genet. 2001; 35: 501-38.

8. Iskow RC, McCabe MT, Mills RE, Torene S, Pittard WS, Neuwald AF, et al. Natural mutagenesis of human genomes by endogenous retrotransposons. Cell. 2010; 141: 1253-61.

9. Rodic N, Sharma R, Sharma R, Zampella J, Dai L, Taylor MS, et al. Long interspersed element-1 protein expression is a hallmark of many human cancers. Am J Pathol. 2014; 184: 1280-6.

10. Brockstedt E, Rickers A, Kostka S, Laubersheimer A, Dorken B, Wittmann-Liebold B, et al. Identification of apoptosis-associated proteins in a human Burkitt lymphoma cell line. Cleavage of heterogeneous nuclear ribonucleoprotein A1 by caspase 3. J Biol Chem. 1998; 273: 28057-64.

11. Yang C, Maiguel DA, Carrier F. Identification of nucleolin and nucleophosmin as genotoxic stress-responsive RNA-binding proteins. Nucleic Acids Res. 2002; 30: 2251-60

12. Abdelmohsen $\mathrm{K}$, Gorospe M. RNA-binding protein nucleolin in disease. RNA Biol. 2012; 9: 799-808.

13. Berger CM, Gaume X, Bouvet P. The roles of nucleolin subcellular localization in cancer. Biochimie. 2015; 113: 78-85.

14. Chalfin HJ, Verdone JE, van der Toom EE, Glavaris S, Gorin MA, Pienta KJ. Nucleolin Staining May Aid in the Identification of Circulating Prostate Cancer Cells. Clin Genitourin Cancer. 2017; 15: e477-e81. PMID: 28153390.

15. Hammoudi A, Song F, Reed KR, Jenkins RE, Meniel VS, Watson AJ, et al. Proteomic profiling of a mouse model of acute intestinal Apc deletion leads to identification of potential novel biomarkers of human colorectal cancer (CRC). Biochem Biophys Res Commun. 2013; 440: 364-70.

16. Pichiorri F, Palmieri D, De Luca L, Consiglio J, You J, Rocci A, et al. In vivo NCL targeting affects breast cancer aggressiveness through miRNA regulation. The Journal of experimental medicine. 2013; 210: 951-68. PMID: 23610125.

17. Xu JY, Lu S, Xu XY, Hu SL, Li B, Li WX, et al. Prognostic significance of nuclear or cytoplasmic nucleolin expression in human non-small cell lung cancer and its relationship with DNA-PKcs. Tumour Biol. 2016; 37: 10349-56.

18. Percharde M, Lin CJ, Yin Y, Guan J, Peixoto GA, Bulut-Karslioglu A, et al. A LINE1-Nucleolin Partnership Regulates Early Development and ESC Identity. Cell. 2018; 174: 391-405 e19.

19. Destouches D, Page N, Hamma-Kourbali Y, Machi V, Chaloin O, Frechault S, et al. A simple approach to cancer therapy afforded by multivalent pseudopeptides that target cell-surface nucleoproteins. Cancer Res. 2011; 71: 3296-305.

20. Reyes-Reyes EM, Aispuro I, Tavera-Garcia MA, Field M, Moore S, Ramos I, et al. LINE-1 couples EMT programming with acquisition of oncogenic phenotypes in human bronchial epithelial cells. Oncotarget. 2017; 8: 103828-42.

21. Bates PI, Choi EW, Nayak LV. G-rich oligonucleotides for cancer treatment. Methods in molecular biology. 2009; 542: 379-92.

22. Reyes-Reyes EM, Ramos IN, Tavera-Garcia MA, Ramos KS. The aryl hydrocarbon receptor agonist benzo(a)pyrene reactivates LINE-1 in HepG2 cells through canonical TGF-beta1 signaling: implications in hepatocellular carcinogenesis. Am J Cancer Res. 2016; 6: 1066-77.

23. De Cola A, Franceschini M, Di Matteo A, Colotti G, Celani R, Clemente E, et al. N6L pseudopeptide interferes with nucleophosmin protein-protein interactions and sensitizes leukemic cells to chemotherapy. Cancer Lett. 2018; 412: $272-82$

24. Destouches D, Huet E, Sader M, Frechault S, Carpentier G, Ayoul F, et al. Multivalent pseudopeptides targeting cell surface nucleoproteins inhibit cancer cell invasion through tissue inhibitor of metalloproteinases 3 (TIMP-3) release. J Biol Chem. 2012; 287: 43685-93. 
25. Gilles ME, Maione F, Cossutta M, Carpentier G, Caruana L, Di Maria S, et al. Nucleolin Targeting Impairs the Progression of Pancreatic Cancer and Promotes the Normalization of Tumor Vasculature. Cancer Res. 2016; 76: 7181-93.

26. Peddigari S, Li PW, Rabe JL, Martin SL. hnRNPL and nucleolin bind LINE-1 RNA and function as host factors to modulate retrotransposition. Nucleic Acids Res. 2013; 41: 575-85.

27. Jia W, Yao Z, Zhao J, Guan Q, Gao L. New perspectives of physiological and pathological functions of nucleolin (NCL). Life Sci. 2017; 186: 1-10.

28. Dhez AC, Benedetti E, Antonosante A, Panella G, Ranieri B, Florio TM, et al. Targeted therapy of human glioblastoma via delivery of a toxin through a peptide directed to cell surface nucleolin. J Cell Physiol. 2018; 233: 4091-105.

29. Diamantopoulou Z, Gilles ME, Sader M, Cossutta M, Vallee B, Houppe C, et al. Multivalent cationic pseudopeptide polyplexes as a tool for cancer therapy. Oncotarget. 2017; 8: 90108-22.

30. Benedetti E, Antonosante A, d'Angelo M, Cristiano L, Galzio R, Destouches D, et al. Nucleolin antagonist triggers autophagic cell death in human glioblastoma primary cells and decreased in vivo tumor growth in orthotopic brain tumor model. Oncotarget. 2015; 6: 42091-104.

31. Jain $\mathrm{N}$, Zhu H, Khashab T, Ye Q, George B, Mathur R, et al. Targeting nucleolin for better survival in diffuse large B-cell lymphoma. Leukemia. 2018; 32: 663-74.

32. Zhang $R$, Zhang $F$, Sun $Z$, Liu $P$, Zhang $X$, Ye $Y$, et al. LINE-1 Retrotransposition Promotes the Development and Progression of Lung Squamous Cell Carcinoma by Disrupting the Tumor Suppressor Gene FGGY. Cancer Res. 2019.

33. Birmpas C, Briand JP, Courty J, Katsoris P. Nucleolin mediates the antiangiogenesis effect of the pseudopeptide N6L. BMC Cell Biol. 2012; 13: 32.

34. Reyes-Reyes EM, Teng Y, Bates PJ. A new paradigm for aptamer therapeutic AS1411 action: uptake by macropinocytosis and its stimulation by a nucleolin-dependent mechanism. Cancer Res. 2010; 70: 8617-29.

35. Bates PJ, Reyes-Reyes EM, Malik MT, Murphy EM, O'Toole MG, Trent JO. G-quadruplex oligonucleotide AS1411 as a cancer-targeting agent: Uses and mechanisms. Biochim Biophys Acta Gen Subj. 2017; 1861: 1414-28.

36. Kabirian-Dehkordi S, Chalabi-Dchar M, Mertani HC, Le Guellec D, Verrier B, Diaz JJ, et al. AS1411-conjugated gold nanoparticles affect cell proliferation through a mechanism that seems independent of nucleolin. Nanomedicine. 2019: 102060 . 